\title{
Long-Term Outcome after Successful Lower Extremity Free Flap Salvage
}

\author{
Amir K. Bigdeli, MD ${ }^{1} \quad$ Emre Gazyakan, MD ${ }^{1} \quad$ Volker J. Schmidt, MD ${ }^{1}$ Christoph Bauer, MD ${ }^{1}$ \\ Günter Germann, MD ${ }^{2}$ Christian A. Radu, MD ${ }^{1} \quad$ Ulrich Kneser, MD ${ }^{1} \quad$ Christoph Hirche, MD ${ }^{1}$
}

1 Department of Hand, Plastic and Reconstructive Surgery, Burn
Center, BG Trauma Center Ludwigshafen, University of Heidelberg,
Ludwigshafen, Germany
${ }^{2}$ Clinic for Plastic, Reconstructive and Aesthetic Surgery, Ethianum
Hospital at University of Heidelberg, Heidelberg, Germany

J Reconstr Microsurg 2019;35:263-269.

\begin{abstract}
Address for correspondence Christoph Hirche, MD, Department of Hand, Plastic and Reconstructive Surgery, Burn Center, BG Trauma Center Ludwigshafen, University of Heidelberg, Ludwig-GuttmannStrasse 13, 67071 Ludwigshafen, Germany (e-mail: christoph.hirche@bgu-ludwigshafen.de).
\end{abstract}

\begin{abstract}
Keywords

- free flap

- revision

- outcome

- anastomosis

- re-exploration
\end{abstract}

As a result of technical advancements and increasing microsurgical experience, free flap surgery has evolved to a routine procedure for reconstruction of complex surgical defects that can be safely employed with success rate up to $99 \%^{1-11}$

received

March 27, 2018

accepted after revision

September 8, 2018

published online

October 16, 2018
However, re-exploration rates up to $15 \%$ are evident in the literature, ${ }^{9,12}$ primarily depending on the location, the underlying cause, and the type of defects. Here, free flap complication rates continue to be higher in lower extremity reconstruction
Copyright $\odot 2019$ by Thieme Medical Publishers, Inc., 333 Seventh Avenue, New York, NY 10001, USA. Tel: +1(212) 584-4662.
DOI https://doi.org/ 10.1055/s-0038-1675146. ISSN 0743-684X. 
compared with other recipient sites. ${ }^{13}$ It is generally accepted that careful monitoring as well as early surgical revision are capable to salvage a microvascular compromised free flap with salvage rates between 43 and $89 \%{ }^{2,6,9,14-16}$ Without any doubt, microvascular complications continue to be a major challenge, and even highly trained microsurgeons are not spared from this potential complication. Nevertheless, no evidence is available on the outcome of salvaged free flaps, which have suffered from temporary tissue hypoxia. There is consensus that urgent reexploration is crucial for flap survival. However, round-theclock availability of a microsurgeon is required, which becomes more and more a logistic challenge in terms of limited human and financial resources even in high volume centers. Comprehensive studies examining the incidences, causes, and methods of avoiding or treating microvascular complications have been performed, but long-term results are scarce. Therefore, the aim of this study was to review our single-center experience and management of a large number of microvascular free flap transfers over an 11-year period with the main emphasis on long-term results after successful lower extremity free flap salvage, a focus that has rarely been addressed before. The analysis of the data may clear up misunderstandings on infrastructural requirements and underline the need for round-theclock availability of a microsurgeon to warrant the highest rate of free flap salvage and hence the reconstructive success.

\section{Methods}

\section{Patients}

The single-center retrospective study including long-term follow-up was approved by the local ethics committee (Mainz, Germany, Reference No. 837.180.11 [7722]). Between January 1999 and December 2010, 581 patients underwent lower extremity reconstruction with a microvascular free flap at the BG Trauma Center Ludwigshafen. The retrospective analysis identified 86 free flaps, which required emergency re-exploration due to microvascular complications. Sixty-five free flaps could be salvaged. Fifteen initially salvaged flaps were excluded from the study because of secondary lower extremity amputation unrelated to flap failure. The hospital records of the remaining 50 patients were reviewed and follow-up obtained in 29 patients (6 females and 23 males) with a mean age of $49.6 \pm 16.6$ years (range: 19-80 years) and mean follow-up time of $54.5 \pm 32.9$ months (range: $10-140$ months). Six patients were lost to follow-up because they had died from causes unrelated to flap surgery. Fifteen patients disagreed to participate in the study or could not be contacted. Written informed consent was obtained from each participating patient. Patient data are summarized in -Table 1 .

\section{Questionnaires}

The Short Form 36 (SF-36) questionnaire was used to assess the patients' postoperative health-related quality of life. ${ }^{17,18}$

\section{Scar Quality}

The aesthetic outcome was analyzed via assessment of the scar quality through the Vancouver Scar Scale (VSS). ${ }^{19,20}$
Table 1 Patient demographics

\begin{tabular}{|l|l|}
\hline Number of free flaps & 581 \\
\hline Number of re-explored free flaps & 86 out of $581(14.8 \%)$ \\
\hline Number of lost free flaps & 31 out of $581(5.3 \%)$ \\
\hline Number of salvaged free flaps & 65 out of $86(75.6 \%)$ \\
\hline Eligible for follow-up & $50(76.9 \%)$ \\
\hline $\begin{array}{l}\text { Number of salvaged free } \\
\text { flaps with follow-up }\end{array}$ & 29 out of $65(58.7 \%)$ \\
\hline Gender (female/male) & $6 / 23$ \\
\hline Age, years (mean; range) & $49.6 \pm 16.6(19-80)$ \\
\hline
\end{tabular}

\section{Management during Re-exploration}

During urgent revision, the flap was raised, and pedicle anatomy and position as well as the site of the anastomosis were carefully analyzed. In any case of reduced blood flow or suspected thrombosis, the anastomosis was reopened and explored. A reanastomosis was performed after resection. If extensive clot formation was identified in the vessel lumen, thrombectomy was performed by milking the vessels with forceps or by small Fogarty catheters, and the flap was rinsed with heparinized saline solution (100 units heparin per milliliter). Afterward, the anastomosis was excised and reanastomosed. When direct reanastomosis was not possible, vein grafts were used. If a purely technical cause of thrombosis without evidence of vascular injury was identified, anastomotic revision to the same recipient vessels was performed. When the recipient vessels were damaged or re-evaluated as insufficient, alternative recipient vessels were dissected or vein grafting was performed. If no systemic contraindications to anticoagulation were present, patients were postoperatively placed on continuous application of intravenous, unfractionated heparin especially when vascular revisions or vein grafts were necessary or clot formation was present. Heparin doses were adjusted to maintain an activated prothrombin time at approximately 2 to 2.5 times normal levels.

\section{Postoperative Monitoring and Flap Conditioning}

The postoperative free flap monitoring protocol consisted of physical examination (swelling, turgor, color, temperature, and "capillary refill"). We always made the effort to harvest a perforator-based or randomized monitor skin paddle within muscle flaps. Acoustic Doppler ultrasound was additionally used in selected flaps, where clinical monitoring was thought to be unreliable. Strict hourly checks were performed by the trained nursing staff for the first 48 postoperative hours, followed by an evaluation of every 2 to 4 hours for the following 3 days. Also, the in-house resident physician performed regular checks (at least every 4 hours). Any abnormality was strictly followed by immediate evaluation by a microsurgeon. When flap circulation was suspected to be compromised, patients were returned immediately to the operating theater.

Postoperatively, a standardized dangling protocol was utilized for flap conditioning starting earliest on the sixth postoperative day after 5 days of strict bed rest and elevation of the lower extremity. Three times per day, the patient's knee was 
bent up without touching the ground for 5 minutes on the first training day. The procedure was supervised by a specially trained nursing staff. The duration of dangling was increased 5 minutes daily and completed by reaching 45 minutes on the ninth postoperative day. Afterward, mobilizing of the patient was started under the assistance of specially trained physical therapists according to the extent of injury of the lower extremity.

\section{Statistical Analysis}

The SPSS software package (version 19.0 for Windows, IBM SPSS Statistics, Inc., Chicago, IL) was used for computerassisted statistical analysis. Descriptive statistics were presented using means with standard deviations or numbers with percentages. Pearson's chi-square test was used to determine the statistical significance of the relationship between re-exploration rate and the studied variables. A pvalue of $<0.05$ was considered statistically significant.

\section{Results}

During the study period, 581 free flap lower extremity reconstructions were performed at our center. Eighty-six free flaps required emergency re-exploration within the first 6 days after surgery due to microvascular complications (arterial and/or venous thrombosis, hematoma with compression of the vascular pedicle), resulting in a re-exploration rate of $14.8 \%$. Free flap salvage was achieved in 65 out of 86 patients, which corresponds to a salvage rate of $75.6 \%$. Thirty-one flaps were totally lost, which corresponds to a total free flap survival rate of $94.7 \%$ and total loss rate of $5.3 \%$. There was no statistically significant correlation between the number of re-explorations and flap loss $(p=1.15)$. Fifteen initially salvaged free flaps were excluded from the follow-up because of secondary amputation of the lower extremity unrelated to flap failure.

Regarding the 50 salvaged free flaps eligible for follow-up, the causes for flap transfer were trauma ( $n=33 ; 66.0 \%)$, infection $(n=11 ; 22.0 \%)$, tumor $(n=1 ; 2.0 \%)$, burn injury $(n=1 ; 2.0 \%)$, radiation ulcer $(n=1 ; 2.0 \%)$, and others $(n=3 ; 6.0 \%)$ as shown in - Fig. 1. There were 11 female patients and 39 male patients with a mean age of $44.0 \pm 15.9$ years (range: $16-74$ years). The mean duration of hospital

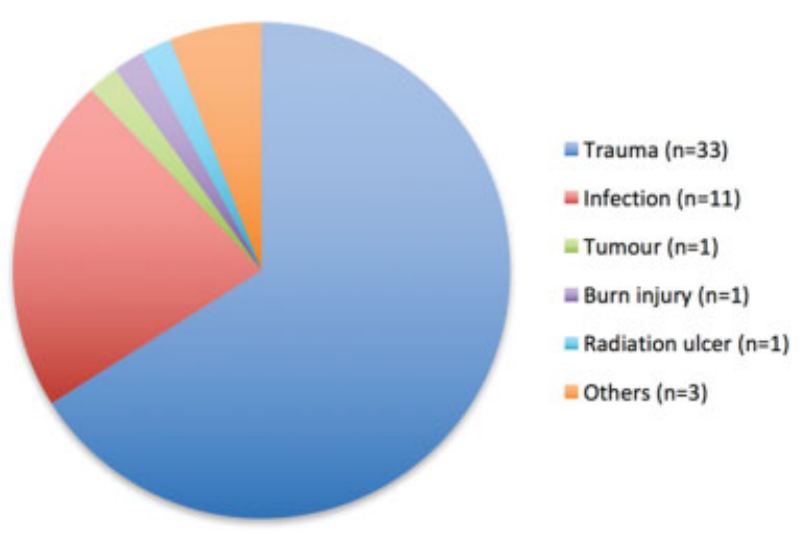

Fig. 1 Indication for free flap transfer in the 50 salvaged flaps eligible for follow-up.
Table 2 Distribution of salvaged free flaps in the 50 patients eligible for follow-up

\begin{tabular}{|l|l|}
\hline Type of flap & Total \\
\hline Anterolateral thigh & 15 \\
\hline Latissimus dorsi & 12 \\
\hline Parascapular & 3 \\
\hline Osteocutaneous scapular/parascapular & 1 \\
\hline Conjoined latissimus dorsi and parascapular & 2 \\
\hline Lateral forearm & 5 \\
\hline Gracilis & 3 \\
\hline Serratus anterior & 1 \\
\hline Combined latissimus dorsi and serratus anterior & 1 \\
\hline Free fibula with skin paddle & 3 \\
\hline Radialis & 1 \\
\hline Transverse rectus abdominis musculocutaneous) & 1 \\
\hline Rectus abdominis & 1 \\
\hline Medial femoral condyle with skin paddle & 1 \\
\hline & 50 \\
\hline
\end{tabular}

stay was $53 \pm 28$ days (range: $20-153$ days). The distribution of the salvaged free flaps is summarized in - Table 2 .

\section{Vascular Imaging}

Regarding the 50 salvaged free flaps eligible for follow-up, vascular imaging of the recipient site was performed in 45 patients prior to free flap surgery (90\%) and showed normal findings in 26 patients (58\%). Fifteen patients (33\%) showed an occlusive disease of the lower extremity with two-vessel flow to the distal leg and four patients (9\%) presented onevessel flow to the distal leg.

\section{Re-exploration}

A total of 66 re-explorations were performed in the 50 salvaged free flaps eligible for follow-up. Causes were dominantly microvascular complications ( $n=46,69.7 \%$ ), followed by hematomas with pedicle compromise $(n=20,30.3 \%)$ (-Fig. 2). Detailed information is given in - Table 3 .

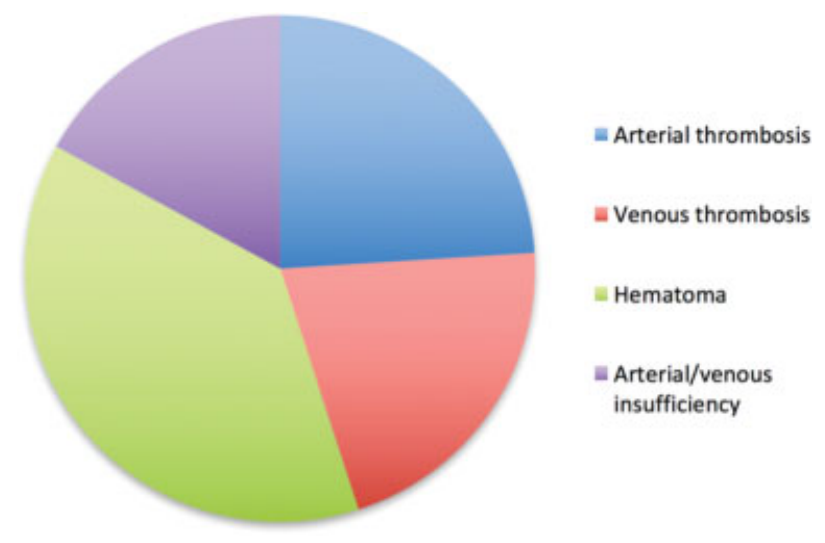

Fig. 2 Causes for free flap re-exploration in the 50 salvaged free flaps eligible for follow-up. 
Table 3 Reasons for free flap re-exploration in the 50 patients eligible for follow-up

\begin{tabular}{|l|l|l|}
\hline Reason for re-exploration & Total & Total (\%) \\
\hline Hematoma & 21 & 32 \\
\hline Arterial thrombosis & 16 & 24 \\
\hline Venous thrombosis & 10 & 15 \\
\hline Arterial and venous thrombosis & 1 & 1.5 \\
\hline Arterial thrombosis and hematoma & 1 & 1.5 \\
\hline Venous thrombosis and hematoma & 1 & 1.5 \\
\hline Arterial insufficiency & 7 & 11 \\
\hline Venous insufficiency & 7 & 11 \\
\hline $\begin{array}{l}\text { Combined arterial and } \\
\text { venous insufficiency }\end{array}$ & 1 & 1.5 \\
\hline $\begin{array}{l}\text { Arterial insufficiency } \\
\text { and hematoma }\end{array}$ & 1 & 1.5 \\
\hline Total & 66 & 100 \\
\hline
\end{tabular}

During initial free flap surgery, single and double venous anastomoses were performed in 33 (66.0\%) and 17 (34.0\%), respectively. Venous compromise alone or in combination was the cause for 17 initial re-explorations (34.0\%), whereas 13 single-venous anastomosis (76.5\%) and 4 double venous anastomosis flaps (23.5\%) were re-explored. Comparison using Pearson's chi-square test showed no statistically significant difference between one and two venous anastomoses regarding the re-exploration rate $(p=0.52)$.

Vein grafting was applied in ten cases for venous compromise and in seven cases for arterial compromise. The median number of re-explorations per patient was one (range: 1-4). Thirty-five free flaps (70.0\%) were re-explored once, 15 patients (30.0\%) underwent two re-explorations, 3 patients (6.0\%) were re-explored three times, and 1 patient (2.0\%) four times. The mean time from the first signs of impaired flap perfusion to re-exploration was $1.3 \pm 0.4$ hours. The mean time from free tissue transfer to the first re-exploration was $16.2 \pm 1.9$ hours. The mean time of re-exploration was $109 \pm 75$ minutes (range: $14-340$ minutes); $67.4 \%$ of all complications, which led to emergency free flap re-exploration, occurred during the first 24 hours after surgery; 20.9\% occurred between 24 and 72 hours and $11.6 \%$ after more than 72 hours postoperatively. During the first 24 hours, the dominant cause for initial re-exploration was arterial thrombosis, followed by venous thrombosis and hematoma with pedicle compromise. Re-exploration between 24 and 72 hours after surgery was dominantly caused by venous thrombosis, followed by hematoma ( - Table 4). After 72 hours, hematoma was the most common cause for re-exploration, whereas all but one patient were postoperatively placed on continuous intravenous heparin and oral aspirin therapy.

Focusing on the 29 salvaged free flaps with follow-up, 34 re-explorations were performed. Causes were dominantly microvascular complications $(n=23,67.6 \%)$, followed by hematoma with pedicle compromise $(n=11,32.4 \%)$.
Table 4 Main reasons for first free flap re-exploration in dependence of the time after free flap transfer of the 50 patients eligible for follow-up

\begin{tabular}{|l|l|l|l|}
\hline \multirow{2}{*}{ Reasons for re-exploration } & \multicolumn{3}{|c|}{$\begin{array}{l}\text { Period (in h) after free flap } \\
\text { transfer and re-exploration }\end{array}$} \\
\cline { 2 - 4 } & $<\mathbf{2 4} \mathbf{~ h}$ & $\mathbf{2 4 - 7 2 ~} \mathbf{~}$ & $>\mathbf{7 2} \mathbf{~ h}$ \\
\hline Arterial thrombosis & 15 & 1 & 0 \\
\hline Venous thrombosis & 9 & 5 & 1 \\
\hline Hematoma & 8 & 4 & 6 \\
\hline
\end{tabular}

Twenty-four free flaps (77.8\%) were re-explored once, five flaps (13.3\%) twice, and one flap (4.4\%) four times. In the last case, venous thrombosis was the cause for three re-explorations, which was attributed to the size mismatch of the venous anastomosis. Bleeding with subsequent hematoma was the cause for the fourth re-exploration and attributed to the aggressive postoperative anticoagulation treatment. However, specific coagulation factors and/or abnormalities were not present.

\section{Consecutive Operations}

Postoperatively, partial flap loss occurred in 10 of 29 flaps (34.5\%). Debridement and successful split-thickness skin grafting was performed during the hospital stay. After hospital discharge, minor wound healing disorders occurred in five patients (17.2\%) and were treated in the same way. Corrective operations were performed in 10 of 29 flaps (34.5\%). Six flaps (20.1\%) were thinned through liposuction and/or excision of abundant tissue. Four flaps were thinned once, one flap twice, and another flap three times. Furthermore, aesthetically disturbing, excessive or instable scars were surgically removed in four cases (13.8\%).

\section{Scar Quality}

According to the VSS, the overall scar appearance was good. The average score was 4.0 points (range: $0-11$ points). The average score for "pigmentation" was 1.4 (range: $0-3$ points) and 1.4 for "pliability" (range: $0-5$ points). Concerning the special parts "scar height" and "vascularity," the average score was 0.6 (0-3 points). At the time of follow-up, two flaps (6.9\%) showed hypertrophic scars. However, there was no keloid formation. Furthermore, the scar at the operative access for re-exploration led to a wider scar and thus worse cosmetic result. Furthermore, once re-explored flaps ( $n=24,82.8 \%$ ) achieved better results concerning the scar quality through the VSS ( 3.8 vs. 5.4 points) when compared with flaps, which were re-explored multiple times $(n=5$, $17.2 \%)$.

\section{Quality of Life}

Mean level of functioning scores for each SF-36 domain was obtained from the 29 patients with follow-up after free flap salvage. In summary, the average physical component score was $54.4 \pm 5.4$ and the mental component score was $63.1 \pm 10.7$ (-Table 5). 
Table 5 SF-36 quality of life scores in the subgroup of 29 patients with follow-up after free flap re-exploration

\begin{tabular}{|l|l|l|l|l|l|}
\hline Subscale & Mean & Median & SD & Minimum & Maximum \\
\hline Physical functioning & 56.2 & 55.0 & 30.3 & 0 & 100 \\
\hline Role limitations (physical problems) & 46.4 & 37.5 & 45.0 & 0 & 100 \\
\hline Bodily pain & 58.0 & 60.5 & 26.3 & 0 & 100 \\
\hline General health & 56.8 & 53.5 & 23.2 & 20.0 & 100 \\
\hline Vitality & 51.4 & 55.0 & 23.5 & 0 & 90.0 \\
\hline Social functioning & 74.3 & 75.0 & 26.5 & 13.0 & 100 \\
\hline Role limitations (emotional problems) & 57.1 & 50.0 & 43.4 & 0 & 100 \\
\hline Mental health & 69.6 & 72.0 & 18.2 & 32.0 & 100 \\
\hline Physical component score & 54.4 & 56.5 & 5.4 & 46.4 & 58.0 \\
\hline Mental component score & 63.1 & 63.4 & 10.7 & 51.4 & 74.3 \\
\hline
\end{tabular}

Abbreviations: SD, standard deviation; SF-36, Short Form 36.

\section{Discussion}

The present study focuses on patients who underwent successful microsurgical re-exploration with consecutive lower extremity free flap salvage, but suffered from a period of diminished or compromised flap perfusion with hypoxia due to temporary vascular compromise. In addition to our discussion of the microsurgical strategy behind this subgroup with successful microvascular revision, the follow-up revealed that these patients neither suffer from reduced scar quality nor from unstable scarring nor from keloid formation. However, patients who underwent microvascular re-exploration once showed better scar quality than patients with multiple re-explorations.

Furthermore, assessment of the patients' quality of life through the SF-36 questionnaire revealed that the patient's quality of life was slightly reduced but good.

It is generally accepted that free flap complication rates are higher in lower extremity reconstruction compared with other recipient sites. ${ }^{13}$ In this field, Culliford et al ${ }^{13}$ presented their experience with a series of 588 lower extremity free flap reconstructions and found an overall failure rate of 8.5 and $9.0 \%$ in trauma patients. ${ }^{21}$ Interestingly, the failure rate for trauma patients decreased from 11.0 to $3.7 \%$ during three decades, which was attributed to a more critical selection of free flap candidates, improved understanding of the physiology of lower extremity trauma and a more sophisticated multidisciplinary team approach. ${ }^{13}$ We reviewed our experience with 581 lower extremity free flap reconstructions over an 11-year period with the main emphasis on long-term results after free flap salvage, a focus that has rarely been addressed before. With an overall failure rate of $5.3 \%$, our experience is conform to that of Culliford et al. ${ }^{13}$ Stranix et al recently compared salvage rates between fasciocutaneous and muscle free flaps for lower extremity trauma reconstruction. ${ }^{21}$ They found that fasciocutaneous flaps underwent earlier and more frequent microvascular re-exploration with higher successful salvage rates. ${ }^{21}$ DeFazio et al contributed their experience with lower extremity free flap reconstruction in high-risk patients with thrombophilia. ${ }^{22}$ They concluded that, despite high success rates, thrombophilia appears to increase the risk of "nonsalvageability" and recommended to take this into account when counseling patients regarding the risks and benefits of microsurgical reconstruction. ${ }^{22}$ Regarding the outcome after successful microsurgical free flap salvage, Disa et al retrospectively reviewed 400 consecutive free flap reconstructions in 396 patients for oncologic surgical defects with an overall success rate of $97.0 \%{ }^{23}$ Twenty-eight flaps (7.0\%) were re-explored with a salvage rate of $61.0 \%$. These results are consistent with our overall free flap success rate of $94.7 \%$ in a microsurgical lower extremity reconstruction. Their main conclusion was that salvaged flaps resulted in a healed wound that did not delay postoperative radiation or chemotherapy. ${ }^{23}$

Hidalgo and Jones reviewed 150 consecutive free tissue transfers to evaluate the role of emergency re-exploration in flap survival. The average time from the first abnormal postoperative control examination to re-exploration was 1.5 hours compared with 1.3 hours in our study. ${ }^{4}$ The investigators concluded that efficacy of clinical monitoring and aggressive re-exploration were responsible for an increase in flap survival from 90 to $98 \% .{ }^{4}$ Our study leads us to share the conclusion that vigilant monitoring and a prospective indication for urgent reexploration is the key to free flap salvage, despite the logistic challenge to provide sufficient resources for emergency interventions in hospitals.

Furthermore, large series have concluded that $80 \%$ of microvascular complications after free flap surgery occurred within the first 2 postoperative days and successful salvage was more likely to occur in the early postoperative period. ${ }^{6,15,24}$ Long-term results of other studies also justify the indication for complex and resource-consuming re-explorations. $^{2,4,6,25}$ The fact that $67.4 \%$ of all complications requiring re-exploration occurred during the first 24 hours after surgery in our study underscores, in our opinion, the importance of close early postoperative monitoring as the ultimate key to immediate free flap salvage. Here, Pohlenz et al retrospectively reviewed the surgical outcome and complications of 1,000 microvascular free flaps in head and neck 
surgery and proposed that microsurgeons should always check everything for themselves and not trust the on-duty doctors and nursing staff blindly, due to the large number of possible errors in flap transplantation. ${ }^{26}$

Many reports have shown that venous thrombosis is a more common complication than arterial thrombosis in free flap surgery. ${ }^{7,24,25}$ Our study revealed a higher number of arterial thrombosis ( 24 vs. $15 \%$ ), which may be attributable to the high rate of reconstructions in traumatized lower extremities. In our study, consistent with other investigations, the dominant cause for re-exploration during the first 24 hours was arterial thrombosis, followed by venous thrombosis and hematoma with pedicle compromise. ${ }^{6}$ Reexploration between 24 and 72 hours after surgery were dominantly caused by venous thrombosis, followed by hematoma and finally arterial thrombosis. Kroll et al retrospectively reviewed a series of 990 free flaps and concluded first, that arterial monitoring is most critical immediately after surgery, and second, that venous monitoring becomes progressively more important on the second postoperative day. ${ }^{6}$ After the second postoperative day, pedicle thrombosis was rare, usually venous and more difficult to correct by emergent re-exploration. The investigators concluded that free flap monitoring should be performed carefully and more frequently during the first 2 to 3 postoperative days. They further concluded that flap monitoring up to 7 days may be beneficial when flap survival is essential to the patient's life or well-being. ${ }^{6}$ Measuring the benefits against the costs of necessary resources, postoperative monitoring of free flaps seems to be justified for the first 2 postoperative days, as the risk for vascular complications decreases significantly afterward. ${ }^{6}$ Henderson et al recently presented their experience with late anastomotic thrombosis on or after postoperative day 5 in 3,212 patients. Twentythree patients $(0.7 \%)$ suffered from late thrombosis. With a salvage rate of $60.8 \%$, they concluded that free flap survival after late thrombosis can be higher than previously reported, underscoring the importance of rigorous postoperative monitoring, as well as the importance of earliest re-exploration. $^{27}$

Numerous studies have also demonstrated that the most critical factor in immediate free flap salvage is the timing of re-exploration and anastomotic revision. ${ }^{4,14,24,28}$ The critical window for successful free flap salvage appears to be within the first 24 to 48 postoperative hours, ${ }^{4,6,29,30}$ which was also seen in our study.

Finally, our study is limited because of the small number of patients and heterogeneity of the patient groups, which is attributable to the fact that vascular occlusion is fortunately a rare complication and successful salvage again reduces the rate of eligible flaps. The small number of patients affects the interpretation of the data, but to our knowledge, there is no study yet published exclusively addressing the long-term follow-up after free flap salvage. The lack of a comparative group and a standardized assessment tool for objective evaluation of the cosmetic outcome after reconstructive surgery are further limitations, which we will address in a future study.

\section{Conclusion}

Consequent monitoring through trained staff and the opportunity of 24h urgent re-exploration are critical for salvage of microvascular compromised free flaps, not only for the immediate but also for long-term outcome. The follow-up of the patients in this study revealed that successful reexploration does not lead to an increased impairment of the wound situation due to temporary hypoxia and ischemia until successful re-exploration. This study provides evidence for a good long-term outcome for the rare group of free flaps after successful salvage due to vascular compromise.

\section{Conflict of Interest}

None of the authors received any funds or has any financial interests to disclose.

\section{References}

1 Shaw WW. Microvascular free flaps. The first decade. Clin Plast Surg 1983;10(01):3-20

2 Khouri RK, Cooley BC, Kunselman AR, et al. A prospective study of microvascular free-flap surgery and outcome. Plast Reconstr Surg 1998;102(03):711-721

3 Pohlenz P, Blessmann M, Blake F, Li L, Schmelzle R, Heiland M. Outcome and complications of 540 microvascular free flaps: the Hamburg experience. Clin Oral Investig 2007;11(01):89-92

4 Hidalgo DA, Jones CS. The role of emergent exploration in freetissue transfer: a review of 150 consecutive cases. Plast Reconstr Surg 1990;86(03):492-498, discussion 499-501

5 Khouri RK. Free flap surgery. The second decade. Clin Plast Surg 1992;19(04):757-761

6 Kroll SS, Schusterman MA, Reece GP, et al. Timing of pedicle thrombosis and flap loss after free-tissue transfer. Plast Reconstr Surg 1996;98(07):1230-1233

7 Kubo T, Yano K, Hosokawa K. Management of flaps with compromised venous outflow in head and neck microsurgical reconstruction. Microsurgery 2002;22(08):391-395

8 Salemark L. International survey of current microvascular practices in free tissue transfer and replantation surgery. Microsurgery 1991;12(04):308-311

9 Ho MW, Brown JS, Magennis P, et al. Salvage outcomes of free tissue transfer in Liverpool: trends over 18 years (1992-2009). Br J Oral Maxillofac Surg 2012;50(01):13-18

10 Xiong L, Gazyakan E, Wähmann M, et al. Microsurgical reconstruction for post-traumatic defects of lower leg in the elderly: a comparative study. Injury 2016;47(11):2558-2564

11 Xiong L, Gazyakan E, Kremer T, et al. Free flaps for reconstruction of soft tissue defects in lower extremity: a meta-analysis on microsurgical outcome and safety. Microsurgery 2016;36(06):511-524

12 Disa JJ, Pusic AL, Hidalgo DH, Cordeiro PG. Simplifying microvascular head and neck reconstruction: a rational approach to donor site selection. Ann Plast Surg 2001;47(04):385-389

13 Culliford AT IV, Spector J, Blank A, Karp NS, Kasabian A, Levine JP. The fate of lower extremities with failed free flaps: a single institution's experience over 25 years. Ann Plast Surg 2007;59 (01):18-21, discussion 21-22

14 Panchapakesan V, Addison P, Beausang E, Lipa JE, Gilbert RW, Neligan PC. Role of thrombolysis in free-flap salvage. J Reconstr Microsurg 2003;19(08):523-530

15 Brown JS, Devine JC, Magennis P, Sillifant P, Rogers SN, Vaughan ED. Factors that influence the outcome of salvage in free tissue transfer. Br J Oral Maxillofac Surg 2003;41(01):16-20

16 Nakatsuka T, Harii K, Asato H, et al. Analytic review of 2372 free flap transfers for head and neck reconstruction following cancer resection. J Reconstr Microsurg 2003;19(06):363-368, discussion 369 
17 Ware JEJ Jr, Sherbourne CD. The MOS 36-item short-form health survey (SF-36). I. Conceptual framework and item selection. Med Care 1992;30(06):473-483

18 Morfeld M, Bullinger M, Nantke J, Brähler E. The version 2.0 of the SF-36 Health Survey: results of a population-representative study [in German]. Soz Praventivmed 2005;50(05):292300

19 Baryza MJ, Baryza GA. The Vancouver Scar Scale: an administration tool and its interrater reliability. J Burn Care Rehabil 1995;16 (05):535-538

20 Sullivan T, Smith J, Kermode J, McIver E, Courtemanche DJ. Rating the burn scar. J Burn Care Rehabil 1990;11(03):256-260

21 Stranix JT, Lee ZH, Jacoby A, et al. Forty years of lower extremity take-backs: flap type influences salvage outcomes. Plast Reconstr Surg 2018;141(05):1282-1287

22 DeFazio MV, Hung RW, Han KD, Bunting HA, Evans KK. Lower extremity flap salvage in thrombophilic patients: managing expectations in the setting of microvascular thrombosis. J Reconstr Microsurg 2016;32(06):431-444

23 Disa JJ, Hu QY, Hidalgo DA. Retrospective review of 400 consecutive free flap reconstructions for oncologic surgical defects. Ann Surg Oncol 1997;4(08):663-669
24 Bui DT, Cordeiro PG, Hu Q-Y, Disa JJ, Pusic A, Mehrara BJ. Free flap reexploration: indications, treatment, and outcomes in 1193 free flaps. Plast Reconstr Surg 2007;119(07):2092-2100

25 Hidalgo DA, Disa JJ, Cordeiro PG, Hu QY. A review of 716 consecutive free flaps for oncologic surgical defects: refinement in donor-site selection and technique. Plast Reconstr Surg 1998;102 (03):722-732, discussion 733-734

26 Pohlenz P, Klatt J, Schön G, Blessmann M, Li L, Schmelzle R. Microvascular free flaps in head and neck surgery: complications and outcome of 1000 flaps. Int J Oral Maxillofac Surg 2012;41(06): 739-743

27 Henderson PW, Fernandez JG, Cemal Y, et al. Successful salvage of late anastomotic thrombosis after free tissue transfer. J Reconstr Microsurg 2016;32(04):316-324

28 Weinzweig N, Gonzalez M. Free tissue failure is not an all-or-none phenomenon. Plast Reconstr Surg 1995;96(03):648-660

29 Wheatley MJ, Meltzer TR. The role of vascular pedicle thrombectomy in the management of compromised free tissue transfers. Ann Plast Surg 1996;36(04):360-364

30 Betancourt FM, Mah ET, McCabe SJ. Timing of critical thrombosis after replantation surgery of the digits. J Reconstr Microsurg 1998;14(05):313-316 